\title{
A NOTE ON BILINEAR FORMS
}

\author{
BY R. E. A. C. PALEY $\dagger$
}

1. Introduction. In this note I consider the proof of the following theorem due to M. Riesz. $\ddagger$

Theorem. Let $M^{*}(\alpha, \gamma)$ denote the maximum for variable $x$ of

$$
\frac{\left(\sum_{n=1}^{N} B_{n}\left|\sum_{m=1}^{M} A_{m n} x_{m}\right|^{1 / \gamma}\right)^{\gamma}}{\left(\sum_{m=1}^{M} C_{m}\left|x_{m}\right|^{1 / \alpha}\right)^{\alpha}}
$$

where the numbers $A_{m n},(1 \leqq m \leqq M, 1 \leqq n \leqq N)$, are fixed and the numbers $B_{n},(1 \leqq n \leqq N)$, and $C_{m},(1 \leqq m \leqq M)$, are all positive. Then $\log M^{*}(\alpha, \gamma)$ is a convex function of the variables $(\alpha, \gamma)$ in the triangle

$$
0 \leqq \gamma \leqq \alpha \leqq 1
$$

of the $(\alpha, \gamma)$ plane.

I show here that it is sufficient to prove the theorem in the case where $\gamma=\alpha,(0 \leqq \alpha \leqq 1)$. Suppose the theorem to have been established in this case.

2. Proof of the Theorem. Let $d_{n},(1 \leqq n \leqq N)$, be a set of positive numbers for which

$$
\sum_{n=1}^{N} B_{n} d_{n}=1
$$

Then, if $\gamma \leqq \alpha$ the numerator of (1) is the maximum, for all sets of $d_{n}$ satisfying (2), of the expression

$$
\left(\sum_{n=1}^{N} B_{n}\left|\sum_{m=1}^{M} A_{m n} x_{m}\right|^{1 / \alpha} d_{n}^{1-\gamma / \alpha}\right)^{\alpha} .
$$

$\dagger$ With profound regret, the Editors note that the author of this paper died on April 3, 1933. This paper therefore appears posthumously, though the author had read the proofs of it.

$\ddagger$ M. Riesz, Sur les maxima des formes bilinéaires, et sur les fonctions linéaires, Acta Mathematica, vol. 49 (1926), pp. 465-497. 
Now on any segment of a line contained strictly in the triangle $0 \leqq \gamma \leqq \alpha \leqq 1,1-\gamma / \alpha$ is of the form $\lambda+\mu / \alpha$, where $\lambda$ and $\mu$ are constants. Substituting in (3), we see that the maximum of (1) is the maximum, for varying $x$ and $d$, of the expression

$$
\left(\frac{\sum_{n=1}^{N} B_{n}{ }^{\prime}\left|\sum_{m=1}^{M} A_{m n}^{\prime} x_{m}\right|^{1 / \alpha}}{\sum_{m=1}^{M} C_{m}\left|x_{m}\right|^{1 / \alpha}}\right)^{\alpha},
$$

where $A_{m n}^{\prime}, B_{n}^{\prime}$ vanish whenever $d_{n}=0$, and otherwise

$$
A_{m n}^{\prime}=A_{m n} d_{n}{ }^{\mu}, B_{n}{ }^{\prime}=B_{n} d_{n}{ }^{\lambda} .
$$

Now assuming the theorem to have been already established in the case where $\gamma=\alpha$, we see that, for each fixed set of $d_{n}$ the logarithm of the maximum of the expression (4) is convex, and hence $\log M^{*}(\alpha, \gamma)$, as the maximum of a set of convex functions, is also convex. This proves the required result.

3. A Correction. I take this opportunity of remarking that a previous paper $\dagger$ of mine, which gives a proof of the theorem of this note, contains an error, which has been pointed out to me independently by Professor Pólya, Professor M. Riesz, and Professor Tamarkin. In fact, in $\$ 2$ of this paper, I assume, quite unjustifiably, that $\log M^{*}(\alpha, \gamma)$ is differentiable, while all that we can legitimately assert is its continuity. The argument can be rectified by some complicated, but rather uninteresting, lemmas on convex functions, but I shall not attempt to carry this out here.

Massachusetts Institute of Technology

$\dagger$ R. E. A. C. Paley, $A$ proof of a theorem on bilinear forms, Journal of the London Mathematical Society, vol. 6 (1931), pp. 226-230. 